\title{
Dirac cones reshaped by interaction effects in suspended graphene
}

\author{
D. C. Elias', R. V. Gorbachev', A. S. Mayorov', S. V. Morozov², A. A. Zhukov , P. Blake3, \\ L. A. Ponomarenko', I. V. Grigorieva' ${ }^{1}$ K. S. Novoselov ${ }^{1}$, F. Guinea ${ }^{4 \star}$ and A. K. Geim ${ }^{1,3}$
}

In graphene, electron-electron interactions are expected to play a significant role, as the screening length diverges at the charge neutrality point and the conventional Landau theory that enables us to map a strongly interacting electronic liquid into a gas of non-interacting fermions is no longer applicable ${ }^{1,2}$. This should result in considerable changes in graphene's linear spectrum, and even more dramatic scenarios, including the opening of an energy gap, have also been proposed ${ }^{3-5}$. Experimental evidence for such spectral changes is scarce, such that the strongest is probably a $20 \%$ difference between the Fermi velocities $v_{F}$ found in graphene and carbon nanotubes ${ }^{6}$. Here we report measurements of the cyclotron mass in suspended graphene for carrier concentrations $\boldsymbol{n}$ varying over three orders of magnitude. In contrast to the single-particle picture, the real spectrum of graphene is profoundly nonlinear near the neutrality point, and $v_{\mathrm{F}}$ describing its slope increases by a factor of more than two and can reach $\approx 3 \times 10^{6} \mathrm{~m} \mathrm{~s}^{-1}$ at $n<10^{10} \mathrm{~cm}^{-2}$. No gap is found at energies even as close to the Dirac point as $\sim 0.1 \mathrm{meV}$. The observed spectral changes are well described by the renormalization group approach, which yields corrections logarithmic in $\boldsymbol{n}$.

In the first approximation, charge carriers in graphene behave like massless relativistic particles with a conical energy spectrum $E=v_{\mathrm{F}} \hbar k$ where the Fermi velocity $v_{\mathrm{F}}$ plays the role of the effective speed of light and $k$ is the wave vector. Because graphene's spectrum is filled with electronic states up to the Fermi energy, their Coulomb interaction has to be taken into account. To do this, the standard approach of Landau's Fermi-liquid theory, proven successful for normal metals, fails in graphene, especially at $E$ close to the neutrality point, where the density of states vanishes. This leads to theoretical divergences that have the same origin as those in quantum electrodynamics and other interacting-field theories. In the latter case, the interactions are normally accounted for by using the renormalization group theory ${ }^{1}$, that is, by defining effective models with a reduced number of degrees of freedom and treating the effect of high-energy excitations perturbatively. This approach was also applied to graphene by using as a small parameter either the effective coupling constant $\alpha=e^{2} / \hbar v_{\mathrm{F}}$ (refs 7,8) or the inverse of the number of fermion species in graphene $N_{\mathrm{f}}=4$ (refs 9,10). The resulting many-body spectrum is shown in Fig. 1.

As for experiment, graphene placed on top of an oxidized Si wafer and with typical $n \approx 10^{12} \mathrm{~cm}^{-2}$ exhibits $\nu_{\mathrm{F}}$ with the conventional value $v_{\mathrm{F}}^{*} \approx 1.05 \pm 0.1 \times 10^{6} \mathrm{~m} \mathrm{~s}^{-1}$. The value was measured by using a variety of techniques including the early transport experiments, in which Shubnikov-de Haas oscillations $(\mathrm{SdHO})$ were analysed to extract $v_{\mathrm{F}}$ (refs 11,12). It has been noted

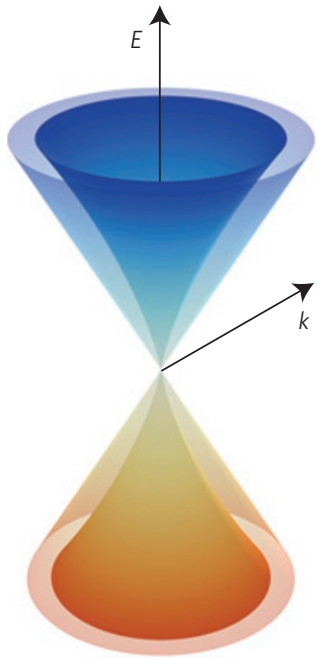

Figure 1 | Sketch of graphene's electronic spectrum with and without taking into account e-e interactions. The outer cone is the single-particle spectrum $E=v_{F} \hbar k$, and the inner cone illustrates the many-body spectrum predicted by the renormalization group theory and observed in the current experiments. We need to consider this image as follows. Electron-electron (e-e) interactions reduce the density of states at low $E$ and lead to an increase in $v_{F}$ that slowly (logarithmically) diverges at zero $E$. As the Fermi energy changes, $v_{F}$ changes accordingly but remains constant under the Fermi surface (note the principal difference from the excitation spectra that probe the states underneath the surface ${ }^{28}$ ).

that $v_{\mathrm{F}}^{*}$ is larger than $v_{\mathrm{F}}^{0} \approx 0.85 \pm 0.05 \times 10^{6} \mathrm{~m} \mathrm{~s}^{-1}$, where $v_{\mathrm{F}}^{0}$ is the value accepted for metallic carbon nanotubes (see, for example, ref. 6). In agreement with this notion, the energy gaps measured in semiconducting nanotubes show a nonlinear dependence on their inverse radii, which is consistent with the larger $v_{\mathrm{F}}$ in flat graphene ${ }^{6}$. The differences between $v_{\mathrm{F}}$ in graphene and its rolled-up version can be attributed to e-e interactions ${ }^{13}$. Another piece of evidence came from infrared measurements ${ }^{14}$ of the Pauli blocking in graphene, which showed a sharp (15\%) decrease in $v_{\mathrm{F}}$ on increasing $n$ from $\approx 0.5$ to $2 \times 10^{12} \mathrm{~cm}^{-2}$. A similar increase in $v_{\mathrm{F}}(\approx 25 \%)$ for similar $n$ has recently been found by scanning tunnelling spectroscopy ${ }^{15}$. In both cases, the changes were sharper and larger than the theory predicts for the probed relatively small intervals of $n$.

Here, we have studied SdHO in suspended graphene devices (inset in Fig. 2a). They were fabricated by using the procedures

\footnotetext{
${ }^{1}$ School of Physics \& Astronomy, University of Manchester, Manchester M13 9PL, UK, ${ }^{2}$ Institute for Microelectronics Technology, 142432 Chernogolovka, Russia, ${ }^{3}$ Manchester Centre for Mesoscience \& Nanotechnology, University of Manchester, Manchester M13 9PL, UK, ${ }^{4}$ Instituto de Ciencia de Materiales de Madrid (CSIC), Sor Juana Inés de la Cruz 3, Madrid 28049, Spain. *e-mail: paco.guinea@icmm.csic.es.
} 


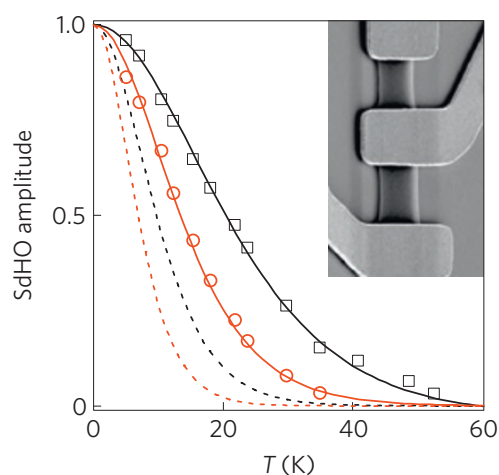

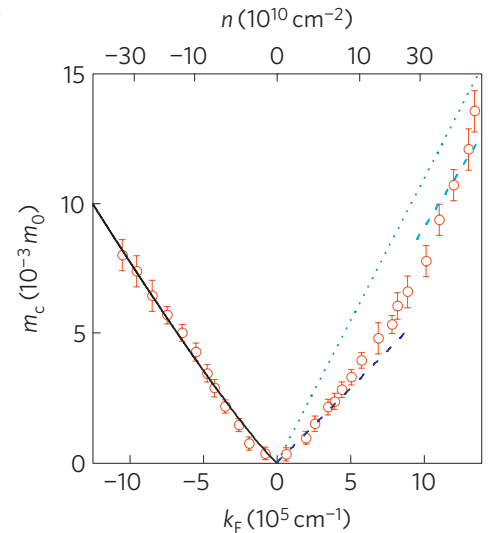

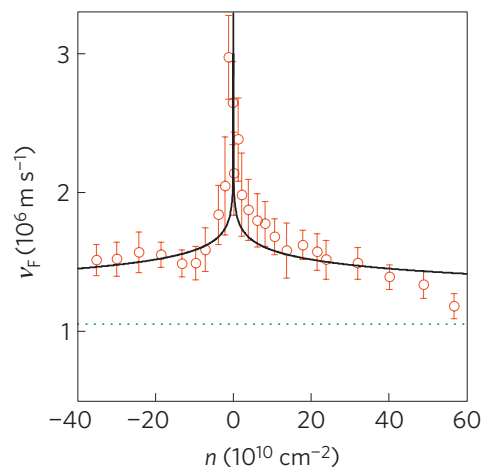

Figure 2 | Probing graphene's electronic spectrum through SdHO. a, Symbols show examples of the $T$ dependence of SdHO for $n \approx+1.4$ and $-7.0 \times 10^{10} \mathrm{~cm}^{-2}$ for electrons and holes, respectively. The dependence is well described by the Lifshitz-Kosevich formula (solid curves). The dashed curves are the behaviour expected for $v_{F}=v_{F}^{*}$ (in the matching colours). The inset shows a scanning electron micrograph of one of our devices. The vertical graphene wire is $\approx 2 \mu \mathrm{m}$ wide and suspended above an oxidized Si wafer attached to Au/Cr contacts. Approximately half of the 300 -nm-thick $\mathrm{SiO} 2$ was etched away underneath the graphene structure. $\mathbf{b}, m_{\mathrm{C}}$ as a function of $k_{\mathrm{F}}$ for the same device. $m_{0}$ is the free-electron mass. It is the exponential dependence of the $\mathrm{SdHO}$ amplitude on $m_{c}$ that enables high accuracy of the cyclotron-mass measurements. The error bars indicate maximum and minimum values of $m_{\mathrm{c}}$ that could fit data such as in $\mathbf{a}$. The dashed curves are the best linear fits $m_{\mathrm{c}} \propto n^{1 / 2}$ at high and low $n$. The dotted line is for the standard value of $v_{F}=v_{F}^{*}$. Graphene's spectrum renormalized owing to e-e interactions is expected to result in the dependence shown by the solid curve. c, $m_{\mathrm{C}}$ re-plotted in terms of varying $v_{\mathrm{F}}$. The colour scheme is to match the corresponding data in $\mathbf{b}$.

described previously ${ }^{16-18}$. After current annealing, our devices exhibited record mobilities $\mu \sim 1,000,000 \mathrm{~cm}^{2} \mathrm{Vs}^{-1}$, and charge homogeneity $\delta n$ was better than $10^{9} \mathrm{~cm}^{-2}$ such that we observed the onset of $\mathrm{SdHO}$ in magnetic fields $B \approx 0.01 \mathrm{~T}$ and the first quantum Hall plateau became clearly visible in $B$ below $0.1 \mathrm{~T}$ (see Supplementary Information). To extract the information about graphene's electronic spectrum, we employed the following routine. SdHO were measured at various $B$ and $n$ as a function of temperature $(T)$. Their amplitude was then analysed by using the standard Lifshitz-Kosevich formula $T / \sinh \left(2 \pi^{2} T m_{c} / \hbar e B\right)$, which holds for the Dirac spectrum ${ }^{19}$ and enables us to find the effective cyclotron mass $m_{c}$ at a given $n$. This approach was previously employed for graphene on $\mathrm{SiO}_{2}$, and it was shown that, within experimental accuracy and for a range of $n \sim 10^{12} \mathrm{~cm}^{-2}, m_{\mathrm{c}}$ was well described by dependence $m_{\mathrm{c}}=\hbar(\pi n)^{1 / 2} / v_{\mathrm{F}}^{*}$, which corresponds to the linear spectrum ${ }^{11,12}$. With respect to the earlier experiments, our suspended devices offer critical advantages. First, in the absence of a substrate, interaction-induced spectral changes are expected to be maximal because no dielectric screening is present. Second, the high quality of suspended graphene has enabled us to probe its spectrum over a very wide range of $n$, which is essential as the spectral changes are expected to be logarithmic in $n$. Third, owing to low $\delta n$, we can approach the Dirac point within a few millielectronvolts. This low- $E$ regime, in which a major renormalization of the Dirac spectrum is expected, has previously been inaccessible.

Figure $2 \mathrm{a}$ shows examples of the $\mathrm{T}$ dependence of the $\mathrm{SdHO}$ amplitude at low $n$ (for details, see Supplementary Information). The curves are well described by the Lifshitz-Kosevich formula but the inferred $m_{\mathrm{c}}$ are half those expected if we assume that $v_{\mathrm{F}}$ retains its conventional value $v_{\mathrm{F}}^{*}$. To emphasize this profound discrepancy with the earlier experiments, the dashed curves in Fig. 2a plot the $T$ dependence expected under the assumption $v_{\mathrm{F}}=v_{\mathrm{F}}^{*}$. The $\mathrm{SdHO}$ would then have to decay twice as fast with increasing $T$, which would result in a qualitatively different behaviour of the SdHO. From the measured $m_{\mathrm{c}}$ we find $v_{\mathrm{F}} \approx 1.9$ and $2.2 \times 10^{6} \mathrm{~m} \mathrm{~s}^{-1}$ for the higher and lower $|n|$ in Fig. $2 \mathrm{a}$, respectively. We have carried out measurements of $m_{\mathrm{c}}$ as in Fig. 2a for many different $n$, and the extracted values are presented in Fig. $2 \mathrm{~b}$ for one of the devices. For the linear spectrum, $m_{\mathrm{c}}$ is expected to increase linearly with $k_{\mathrm{F}}=(\pi n)^{1 / 2}$. In contrast, the experiment shows a superlinear behaviour. Trying to fit the curves in Fig. $2 \mathrm{~b}$ with the linear dependence $m_{\mathrm{c}}\left(k_{\mathrm{F}}\right)$, we find $v_{\mathrm{F}} \geq 2.5 \times 10^{6} \mathrm{~m} \mathrm{~s}^{-1}$ at $n<10^{10} \mathrm{~cm}^{-2}$ and $\leq 1.5 \times 10^{6} \mathrm{~m} \mathrm{~s}^{-1}$ for $n>2 \times 10^{11} \mathrm{~cm}^{-2}$, as indicated by the dashed lines. The observed superlinear dependence of $m_{\mathrm{c}}$ can be translated into $v_{\mathrm{F}}$ varying with $n$. Figure $2 \mathrm{c}$ replots the data in Fig. $2 \mathrm{~b}$ in terms of $v_{\mathrm{F}}=\hbar(\pi n)^{1 / 2} / m_{\mathrm{c}}$, which shows a diverging-like behaviour of $v_{\mathrm{F}}$ near the neutrality point. This sharp increase in $v_{\mathrm{F}}$ (by nearly a factor of three with respect to $v_{\mathrm{F}}^{*}$ ) contradicts to the linear model of graphene's spectrum but is consistent with the spectrum reshaped by e-e interactions (Fig. 1).

The data for $m_{\mathrm{c}}$ measured in four devices extensively studied in this work are collected in Fig. 3 and plotted on a logarithmic scale for both electrons and holes (no electron-hole asymmetry was noticed). The plot covers the experimental range of $|n|$ from $10^{9}$ to nearly $10^{12} \mathrm{~cm}^{-2}$. All the data fall within the range marked by the two dashed curves that correspond to constant $v_{\mathrm{F}}=v_{\mathrm{F}}^{*}$ and $v_{\mathrm{F}}=3 \times 10^{6} \mathrm{~m} \mathrm{~s}^{-1}$. We can see a gradual increase in $v_{\mathrm{F}}$ as $n$ increases, although the logarithmic scale makes the observed threefold increase less dramatic than in the linear presentation of Fig. 2c. Note that, even for the highest $n$ in Fig. 3, the measured $m_{\mathrm{c}}$ do not reach the values expected for $v_{\mathrm{F}}=v_{\mathrm{F}}^{*}$ and are better described by $v_{\mathrm{F}} \approx 1.3 v_{\mathrm{F}}^{*}$. This could be due to the fact that the highest $n$ values we could achieve for suspended graphene were still within a sub- $10^{12} \mathrm{~cm}^{-2}$ range, in which some enhancement in $v_{\mathrm{F}}$ was reported for graphene on $\mathrm{SiO}_{2}$ (refs 14,15). Alternatively, the difference could be due to the absence of a substrate in our case. To find out which of the effects dominates, we have studied high- $\mu$ devices made from graphene deposited on boron nitride ${ }^{20,21}$ (its dielectric constant $\varepsilon$ is close to that of $\mathrm{SiO}_{2}$ ) and found that $m_{\mathrm{c}}$ in the range of $n$ between $\approx 0.1$ and $1 \times 10^{12} \mathrm{~cm}^{-2}$ is well described by $v_{\mathrm{F}} \approx v_{\mathrm{F}}^{*}$ (Supplementary Information). This indicates that the observed difference in $m_{\mathrm{c}}$ at high $n$ in Fig. 3 with respect to the values expected for $v_{\mathrm{F}}^{*}$ is likely to be due to the absence of dielectric screening in suspended graphene, which maximizes the interaction effects.

To explain the observed changes in $v_{\mathrm{F}}$, let us first note that, in principle, not only e-e interactions but also other mechanisms such as electron-phonon coupling and disorder can lead to changes in $v_{\mathrm{F}}$. However, the fact that the increase in $v_{\mathrm{F}}$ is observed over such a wide range of $E$ rules out electron-phonon mechanisms, whereas 


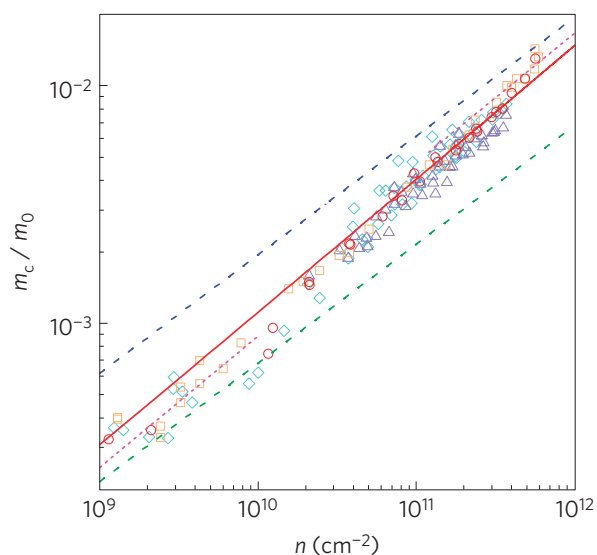

Figure 3 | Interaction-induced changes in the cyclotron mass. Different symbols are the measurements for different devices. The random scatter characterizes the statistical uncertainty for different samples and experiments. Blue and green dashed lines are the behaviour expected for the linear spectrum with constant $v_{F}$ equal to $v_{F}^{*}$ and $3 \times 10^{6} \mathrm{~m} \mathrm{~s}^{-1}$, respectively. The solid red curve is for the spectrum renormalized by e-e interactions and described by equation (2) that takes into account the intrinsic screening self-consistently. The two dotted curves show that the interaction effects can also be described by a simpler theory (equation (1)) with an extra fitting parameter $\varepsilon_{G}(n)$, graphene's intrinsic dielectric constant. The best-fit curves yield $\varepsilon_{\mathrm{G}} \approx 2.2$ and 4.9 at low and high ends of the $n$ range.

the virtual absence of disorder in our suspended graphene makes the influence of impurities also unlikely. Therefore, we focus on e-e interactions, in which case graphene's spectrum is modified as shown in Fig. 1 and, in the first approximation, can be described by two related equations ${ }^{8-10}$,

$$
\begin{gathered}
\frac{k}{v_{\mathrm{F}}} \frac{\partial v_{\mathrm{F}}}{\partial k}=-\frac{e^{2}}{4 \varepsilon \hbar v_{\mathrm{F}}} \\
\frac{k}{v_{\mathrm{F}}} \frac{\partial v_{\mathrm{F}}}{\partial k}=-\frac{8}{\pi^{2} N_{\mathrm{f}}}\left[1-\frac{4 \hbar v_{\mathrm{F}} \varepsilon}{N_{\mathrm{f}} e^{2}}+\frac{8 \hbar v_{\mathrm{F}} \arccos \left(\frac{\pi N_{\mathrm{f}} e^{2}}{8 \hbar v_{\mathrm{F}} \varepsilon}\right)}{N_{\mathrm{f}} e^{2} \pi \sqrt{1-\left(\frac{\pi N_{\mathrm{f}} e^{2}}{8 \hbar v_{\mathrm{F}} \varepsilon}\right)^{2}}}\right]
\end{gathered}
$$

where $\varepsilon=\left(1+\varepsilon_{\mathrm{s}}\right) / 2$ describes the effect of a substrate with a dielectric constant $\varepsilon_{\mathrm{s}}$. Equation (1) can be considered as the leading term in the renormalization group theory expansion in powers of $\alpha=e^{2} / \varepsilon \hbar v_{\mathrm{F}}$, whereas (2) corresponds to a similar expansion in powers of $1 / N_{\mathrm{f}}$ (refs $\left.8-10\right)$. The diagrams that depict these approximations are given in Supplementary Information. Importantly, equation (2) includes self-consistently the screening by graphene's charge carriers. An approximate scheme to incorporate this intrinsic screening while keeping the simplicity of equation (1) is to define an effective screening constant $\varepsilon_{\mathrm{G}}(n)$ for the graphene layer and add it to $\varepsilon$ (for suspended graphene $\varepsilon=\varepsilon_{\mathrm{G}}$ ). Then, integrating equation (1), we obtain the logarithmic dependence ${ }^{8}$

$$
v_{\mathrm{F}}(n)=v_{\mathrm{F}}\left(n_{0}\right)\left[1+\frac{\alpha}{8 \varepsilon_{\mathrm{G}}} \ln \left(n_{0} / n\right)\right]
$$

where $n_{0}$ is the concentration that corresponds to the ultraviolet cutoff energy $\Lambda$, and $v_{\mathrm{F}}\left(n_{0}\right)$ is the Fermi velocity near the cutoff. We assume $v_{\mathrm{F}}\left(n_{0}\right) \equiv v_{\mathrm{F}}^{0}$, its accepted value in graphene structures with weak e-e interaction.

Both approximations result in a similar behaviour of $v_{\mathrm{F}}(n)$ and provide good agreement with the experiment. However, equation (2) is more general and essentially requires no fitting parameters because $\Lambda$ is expected to be of the order of graphene's bandwidth and affects the fit only weakly, as $\log (\Lambda)$. Alternatively, $\Lambda$ can be estimated from the known value of $v_{\mathrm{F}}^{0}$ at high $n \approx$ $5 \times 10^{12} \mathrm{~cm}^{-2}$ as $\Lambda=2.5 \pm 1.5 \mathrm{eV}$ (ref. 22). The solid curves in Figs $2 \mathrm{~b}, \mathrm{c}$ and 3 show $m_{\mathrm{c}}(n)$ and $v_{\mathrm{F}}(n)$ calculated by integrating equation (2) and using $\Lambda \approx 3 \mathrm{eV}$. The dependence captures all the main features of the experimental data. As for equations (1) and (3), they enable a reasonable fit by using $\varepsilon_{\mathrm{G}} \sim 3.5$ over the whole range of our $n$. More detailed analysis (dotted curves in Fig. 3) yields $\varepsilon_{\mathrm{G}} \approx 2.2$ and 5 for $n \sim 10^{9}$ and $10^{12} \mathrm{~cm}^{-2}$, respectively. These values are close to those calculated in the random phase approximation, which predicts $\varepsilon_{\mathrm{G}}=1+\pi N_{\mathrm{f}} e^{2} / 8 \hbar v_{\mathrm{F}}$. Using this expression in combination with equation (3) leads to a fit that is practically indistinguishable from the solid curve given by equation (2). This could be expected because equation (2) includes the screening self-consistently, also within the random phase approximation. The value of $\varepsilon_{\mathrm{G}}$ has recently become a subject of considerable debate $\mathrm{e}^{23-27}$. Our data clearly show no anomalous screening, contrary to the recent report ${ }^{27}$ that suggested $\varepsilon_{\mathrm{G}} \approx 15$, but in good agreement with measurements reported in ref. 28 .

Finally, a large number of theories have been predicting that the diverging contribution of e-e interactions at low $E$ may result in new electronic phases ${ }^{28-31}$, especially in the least-screened case of suspended graphene with $\varepsilon=1$. Our experiments shows the diverging behaviour of $v_{\mathrm{F}}$ but no new phases emerge, at least for $n>10^{9} \mathrm{~cm}^{-2}(E>4 \mathrm{meV})$. Moreover, we can also conclude that there are no insulating phases even at $E$ as low as $0.1 \mathrm{meV}$. To this end, we refer to Supplementary Information, in which we present the data for graphene's resistivity $\rho(n)$ in zero $B$. The peak at the neutrality point continues to grow monotonically down to $2 \mathrm{~K}$, and $\rho(T)$ exhibits no sign of diverging (the regime of smearing by spatial inhomogeneity is not reached even at this $T$ ). This shows that, in neutral graphene in zero $B$, there is no gap larger than $\approx 0.1 \mathrm{meV}$. This observation is consistent with the fact that $v_{\mathrm{F}}$ increases near the neutrality point, which leads to smaller and smaller $\alpha=e^{2} / \hbar v_{\mathrm{F}}$ at low $E$ and, consequently, prevents the emergence of the predicted many-body gapped states.

Received 1 April 2011; accepted 17 June 2011; published online 24 July 2011; corrected online 21 December 2011

\section{References}

1. Shankar, R. Renormalization-group approach to interacting fermions. Rev. Mod. Phys. 66, 129-192 (1994).

2. Kotov, V. N., Pereira, V. M., Castro Neto, A. H. \& Guinea, F. Electron-electron interactions in graphene: Current status and perspectives. Preprint at http://arxiv.org/abs/1012.3484 (2010).

3. Khveshchenko, D. V. Ghost excitonic insulator transition in layered graphite. Phys. Rev. Lett. 87, 246802 (2001).

4. Gorbar, E. V., Gusynin, V. P., Miransky, V. A. \& Shovkovy, I. A. Magnetic field driven metal-insulator phase transition in planar systems. Phys. Rev. B 66, 045108 (2002).

5. Drut, J. E. \& Lahde, T. A. Is graphene in vacuum an insulator? Phys. Rev. Lett. 102, 026802 (2009).

6. Liang, W. J. et al. Fabry-Perot interference in a nanotube electron waveguide. Nature 411, 665-669 (2001).

7. Abrikosov, A. A. \& Beneslavskii, D. Possible existence of substances intermediate between metals and dielectrics. Sov. Phys. JETP 32, 699-703 (1971).

8. Gonzalez, J., Guinea, F. \& Vozmediano, M. A. H. Non-Fermi liquid behavior of electrons in the half-filled honeycomb lattice (a renormalization group approach). Nucl. Phys. B 424, 595-618 (1994).

9. Gonzalez, J., Guinea, F. \& Vozmediano, M. A. H. Marginal-Fermi-liquid behavior from two-dimensional Coulomb interaction. Phys. Rev. B 59, 2474-2477 (1999).

10. Foster, M. S. \& Aleiner, I. L. Graphene via large N: A renormalization group study. Phys. Rev. B 77, 195413 (2008).

11. Novoselov, K. S. et al. Two-dimensional gas of massless Dirac fermions in graphene. Nature 438, 197-200 (2005). 
12. Zhang, Y. B., Tan, Y. W., Stormer, H. L. \& Kim, P. Experimental observation of the quantum Hall effect and Berry's phase in graphene. Nature 438, 201-204 (2005).

13. Kane, C. L. \& Mele, E. J. Electron interactions and scaling relations for optical excitations in carbon nanotubes. Phys. Rev. Lett. 93, 197402 (2004).

14. Li, Z. Q. et al. Dirac charge dynamics in graphene by infrared spectroscopy. Nature Phys. 4, 532-535 (2008)

15. Luican, A., Li, G. \& Andrei, E. Y. Quantized Landau level spectrum and its density dependence. Phys. Rev. B 83, 041405 (2011).

16. Du, X., Skachko, I., Barker, A. \& Andrei, E. Y. Approaching ballistic transport in suspended graphene. Nature Nanotech. 3, 491-495 (2008).

17. Bolotin, K. I. et al. Ultrahigh electron mobility in suspended graphene. Solid State Commun. 146, 351-355 (2008).

18. Castro, E. V. et al. Limits on charge carrier mobility in suspended graphene due to flexural phonons. Phys. Rev. Lett. 105, 266601 (2010).

19. Sharapov, S. G., Gusynin, V. P. \& Beck, H. Magnetic oscillations in planar systems with the Dirac-like spectrum of quasiparticle excitations. Phys. Rev. B 69, 075104 (2004).

20. Dean, C. R. et al. Boron nitride substrates for high-quality graphene electronics. Nature Nanotech. 5, 722-726 (2010).

21. Abanin, D. A. et al. Giant nonlocality near the Dirac point in graphene. Science 332, 328-330 (2011).

22. de Juan, F., Grushin, A. G. \& Vozmediano, M. A. H. Renormalization of Coulomb interaction in graphene: Determining observable quantities. Phys. Rev. B 82, 125409 (2010).

23. Barlas, Y. et al. Chirality and correlations in graphene. Phys. Rev. Lett. 98, 236601 (2007).

24. Hwang, E. H., Hu, B. Y. K. \& Sarma, S. Density dependent exchange contribution to compressibility in graphene. Phys. Rev. Lett. 99, 226801 (2007).

25. Sheehy, D. E. \& Schmalian, J. Quantum critical scaling in graphene. Phys. Rev. Lett. 99, 226803 (2007).
26. Kotov, V. N., Uchoa, B. \& Neto, A. H. C. Electron-electron interactions in the vacuum polarization of graphene. Phys. Rev. B 78, 035119 (2008).

27. Reed, J. P. et al. The effective fine-structure constant of freestanding graphene measured in graphite. Science 330, 805-808 (2010).

28. Bostwick, A. et al. Observation of plasmarons in quasi-free-standing doped graphene. Science 328, 999-1002 (2010).

29. Khveshchenko, D. V. Ghost excitonic insulator transition in layered graphite. Phys. Rev. Lett. 87, 246802 (2001).

30. Gorbar, E. V., Gusynin, V. P., Miransky, V. A. \& Shovkovy, I. A. Magnetic field driven metal-insulator phase transition in planar systems. Phys. Rev. B 66, 045108 (2002).

31. Drut, J. E. \& Lähde, T. A. Is graphene in vacuum an insulator? Phys. Rev. Lett. 102, 026802 (2009)

\section{Acknowledgements}

This work was supported by the Engineering and Physical Sciences Research Council (UK), the Royal Society, the Air Force Office of Scientific Research, the Office of Naval Research and the Körber Foundation.

\section{Author contributions}

D.C.E., A.S.M., S.V.M.: measurements and data analysis. R.V.G., A.A.Z., P.B.: device fabrication. F.G., A.K.G.: writing up. All the authors contributed to discussions. D.C.E. and R.V.G. contributed to the work equally.

\section{Additional information}

The authors declare no competing financial interests. Supplementary information accompanies this paper on www.nature.com/naturephysics. Reprints and permissions information is available online at http://www.nature.com/reprints. Correspondence and requests for materials should be addressed to F.G. 


\section{CORRIGENDUM}

\section{Dirac cones reshaped by interaction effects in suspended graphene}

D. C. Elias, R. V. Gorbachev, A. S. Mayorov, S. V. Morozov, A. A. Zhukov, P. Blake, L. A. Ponomarenko, I. V. Grigorieva, K. S. Novoselov, F. Guinea and A. K. Geim

Nature Physics 7, 701-704 (2011); published online 24 July 2011; corrected after print 21 December 2011.

In the version of this Letter originally published, the prefactor in equation (2) should have been $-8 / \pi^{2} N_{\mathrm{f}}$. This error has been corrected in the HTML and PDF versions of the Letter. 